\title{
Tygerberg Hospital keeps more hearts beating with pioneering service
}

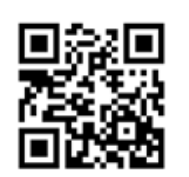

A pioneering life- and costsaving radial angiography service in Tygerberg Hospitals cardiology department, the only one of its kind in Africa, is giving hundreds more patients unprecedented access to accurate diagnosis and treatment.

Built around medical advances that allow both angiography and the often ensuing strategic placement of coronary stents to be done via the radial artery in the arm instead of the traditional and more risky femoral artery in the groin, the custom-built, six-seater radial angiography lounge has helped reduce procedure and recovery times. Every year this gives more than 200 extra referred patients access to diagnostic and therapeutic procedures that could potentially save their lives - and saves the hospital an estimated ZAR1.5 million in what would otherwise have been overnight accommodation, food and linen costs. Private hospitals have already shown interest in emulating both the facility and the service, while the more proactive medical aids are fast sitting up and paying attention.

\section{Patient diagnostic, treatment and discharge times halved}

Tygerberg Hospital cardiologist and chair of the pivotal philanthropic SUNHEART Foundation Dr Alfonso Pecoraro says that the radial angiography suite looks like a dialysis unit but enables patients to undergo and recover from a diagnostic angiogram in 3 hours, and from a stent in 6 . 'The suite is right alongside the theatre, so they literally walk in, get on the table to have the procedures done, and walk

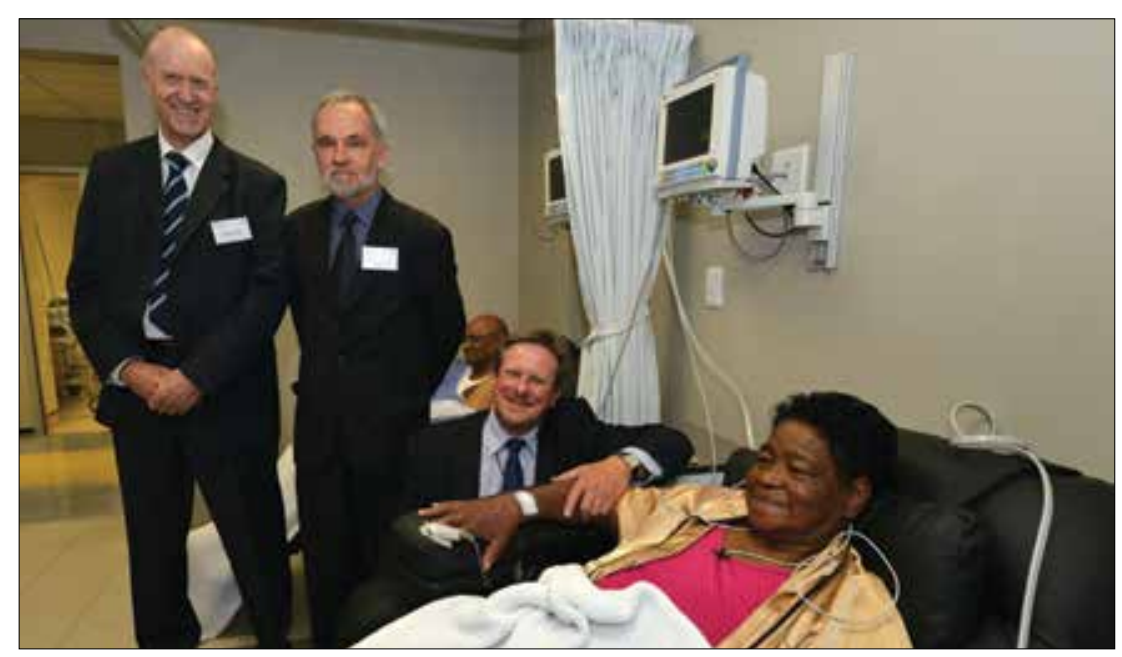

Medtronic MD Mike Howe-Ely, SUNHEART Director Anton Doubell, former Western Cape Minister of Health Theuns Botha and patient Caureen Amelia Petersen.

out. Patients can read or use their laptops to keep occupied if they have nothing else to do while we prep them before or monitor them afterwards,' he said. The product of SUNHEART and private investment partner Medtronic, the suite should increase throughput of patients by approximately $15 \%$ (the cardiology unit performs about 1700 procedures per annum). SUNHEART is the brainchild of Tygerberg's five cardiologists, Charles Kyriakakis, Pecoraro, Anton Doubell, Hellmuth Weich and Philip Herbst, whose ambition it is to change the cardiology landscape by creating access to 'advanced cardiac care for all' through innovative measures and procedures. Medtronic is a Minneapolis (USA)-based global leader in medical technology.
General cardiology awareness and training is also being spread to several of the Western Cape's more rural district hospitals via cardiology satellite outreach facilities that enable staff to be trained in basic cardiology and proper diagnostic work-ups, resulting in a dramatic decline in inappropriate referrals to Tygerberg Hospital. Says Pecoraro: 'The net amount of patients we see has increased, but it's mitigated by the faster turn-around and correct patient profile. Our training commitments have also increased, but hopefully the savings we show will result in more funding. He said that with the World Health Organization predicting that diseases of lifestyle in Africa will have overtaken HIV/AIDS as the leading cause of death within 15 years, the radial angiography 
and stent service was 'massively necessary'. 'As we move towards the Western diet and the Western way of living, South African (SA) statistics on debilitating heart conditions are soaring while they are stabilising in the Western world.' According to Prof. Doubell, Head of the Tygerberg Cardiology Division and Director of SUNHEART, more than 50000 patients in SA annually require advanced cardiac care such as coronary angiography. 'As we shift to a valuebased healthcare culture, the need for innovative solutions that result in high-quality, costeffective healthcare is more important than ever before', he added. SUNHEART's first sponsored research project, the screening of large numbers of schoolchildren for rheumatic heart disease, was launched last year in collaboration with the British Society of Echocardiography. 'More than 1500 children have been screened for rheumatic heart disease during 2014, says Herbst, study leader for Echo in Africa.

\section{Training for proper referral vital}

Pecoraro said that rural cardiovascular training platforms, supported by radial angiography suites in major referral hospitals, had proved themselves in Canada, where the population was 'also geographically challenged'. He said that SUNHEART was already helping several private hospital executives with ideas on planning their own suites and that he believed it would not be long before the big healthcare funders 'insist that this is the way it should be done' Stents had revolutionised cardiovascular care. 'For the right patient it works very well, complication rates are low and usually there's a very good outcome.

Dr Jonny Broomberg, CEO of Discovery Health, said it was inspiring to see this type of innovation and a strong focus on value and patient centeredness in our public healthcare system. We hope this example can be followed by other public and private hospitals, especially if this technique continues to enhance clinical outcomes, reduce cost and improve affordability and cost-effectiveness.

Tygerberg's cardiology division is widely considered a premier training facility for young cardiologists, from both SA and the rest of the African continent. The renovation of the unit's lecture room has created an ultra-modern lecture facility, boosting teaching and training activities in the unit further. An additional fully funded training fellowship has also contributed to an increase in the trainee output from the unit.

\section{Chris Bateman}

chrisb@hmpg.co.za

S Afr Med J 2015;105(5):338-339.

DOI:10.7196/SAMJ.9555 